\title{
Massive Splenomegaly: A Case Report of Visceral Leishmania and Schistosomiasis Co-infection and a Review of Infectious Causes of Massive Splenomegaly
}

\author{
Yael Paran* \\ Head of Travel Medicine Clinic, Tel Aviv Sourasky Medical Center, Israel
}

Massive splenomegaly is typically defined as the lower pole of the spleen extending into the pelvis or crossing the midline or weighing $\geq$ 1000 grams. Non- HIV infectious etiologies of massive splenomegaly narrow the differential diagnosis to Visceral Leishmaniasis (VL or Kala-azar), Schistosomiasis and Hyperreactive Malarial Splenomegaly Syndrome (Tropic Splenomegaly Syndrome). Here we review a case of co-infection with schistosomiasis and VL with massive splenomegaly, few of which have been documented and only studied to date on animal models. We believe this is the first documented case of human co- infection in Israel. We additionally aim to further clarify the presentations and causes of infectious massive splenomegaly.

\section{Case Presentation}

A 29 year- old male Eritrean refugee arrived to Israel one month before hospital presentation. The individual left Eritrea a year earlier, spent nine months in a refugee camp in Ethiopia and then traveled through Sudan and Egypt to reach Israel. He began having drenching night sweats and weight loss during this time. Once in Israel he began to suffer from severe left upper quadrant pain, which made him seek medical advice. He did not report any fever or chills. On presentation his temperature was 37.3 degrees Celsius and he was hemo-dynamically stable with no respiratory distress. Main findings upon physical examination were cachexia, paleness, bilateral axillary lymphadenopathy and a massively enlarged spleen palpable more than $15 \mathrm{~cm}$ below costal margins. There were no signs of cirrhosis. Laboratory test results showed pancytopenia, mild lymphocytosis and an elevated CRP level without eosinophilia (Table 1). Kidney, LFT, $\mathrm{LDH}$ and chest $\mathrm{x}$ - ray was normal. An abdominal CT confirmed the presence of a massive spleen, which was more than $22 \mathrm{~cm}$ in diameter and pushing away the structures of the upper left abdomen (Figure 1). The organ additionally showed an irregular consistency with several stake- shaped, hypodense lesions presumed to be infarcts. There was no pathology in other abdominal organs. The liver was normal in size and showed normal and homogenous consistency with no signs of fibrosis. There were no signs of portal hypertension.

On his first day in the hospital a stool sample for ova and parasites showed ova of Schistosoma mansoni. Although chronic infection with S. Mansoni may cause massive splenomegaly, the history of night sweats and weight loss are not consistent with the diagnosis of schistosomiasis. Additionally, the lack of significant signs of portal hypertension and pipe- stem fibrosis of the liver on the CT support the premise of an alternative or additional cause of the massive splenomegaly.

The patient denied any recurrent febrile illness such as malaria. Blood smear showed $61 \%$ neutrophils, $18 \%$ band cells, relative monocytosis (16\%), microcytosis, macrocytosis, hypochromia and anisocytosis. There was mild hypoalbuminemia (32 gr/l, normal range 35-50) and hypergammaglobulinemia of $50 \mathrm{gr} / \mathrm{l}$ (upper limit 35). Quantitative immunoglobulins showed that the hypergammaglobulinemia was mainly due to elevated IgG levels (35.1 $\mathrm{gr} / \mathrm{l}$, upper limit 16). Electrophoresis of serum protein showed no $\mathrm{M}$ spike. The patient was negative for HIV. An additional abdominal ultrasound showed no signs of periportal liver fibrosis, but did confirm an enlarged spleen of $24.5 \mathrm{~cm}$ with small, focal lesions of $1.5 \mathrm{~cm}$. The ultrasound additionally showed an enlarged liver of $16 \mathrm{~cm}$ in span with homogenous consistency. A bone marrow aspirate showed active trilineage hematopoiesis with mild eosinophilia and no evidence of granuloma or lymphoma. Tests for diagnosis of Leishmania were sent to the national reference laboratory and results were positive for Leishmania antibodies in serum. Additionally, Leishmania from bone marrow aspirate grew in culture and PCR from bone marrow was positive for Leishmania donovani complex.

The patient was diagnosed with visceral Leishmania and coinfecion with Schistosomiasis. He was treated with Praziquantel and liposomal Amphotericin B. The pancytopenia improved under treatment and there was regression in size of the spleen as evidenced by physical examination and in a repeated abdominal CT 6 weeks after the end of treatment (Table 1 and Figure 2).

\section{Discussion}

Massive splenomegaly has a narrow differential diagnosis, which includes several hematological conditions such as chronic myeloid leukemia, lymphoma, myelofibrosis, thalassemia major, Gaucher's disease and infectious etiologies [1]. In a non- HIV patient there are only three infectious causes of massive splenomegaly: VL, Schistosomiasis and Hyperreactive Malaria Syndrome. The clinical and laboratory presentation of each is listed below (Table 2).

The most important clinical manifestation of VL is the syndrome known as kala- azar (Hindi for "black fever"). The incubation period is usually 2-6 months but can range from a few weeks to several years. Onset of symptoms is usually insidious or subacute with slow progression of malaise, fever, weight loss and splenomegaly (with or without hematomegaly) over a period of months. The

${ }^{*}$ Correspondence to: Yael Paran, Head of Travel Medicine Clinic, Tel Aviv Sourasky Medical Center, Israel, Tel: +972-52-4262723, +972-3-6974345; Fax: +972-3-6976696; E-mail: yaelp@tlvmc.gov.il

Received: June 19, 2018; Accepted: June 23, 2018; Published: June 30, 2018 
Paran Y (2018) Massive Splenomegaly: A Case Report of Visceral Leishmania and Schistosomiasis Co-infection and a Review of Infectious Causes of Massive Splenomegaly

Table 1. Patient Lab Values

\begin{tabular}{|c|c|c|c|}
\hline & On Admission & Before Treatment \\
\hline Hemoglobin $(13.5-17.5 \mathrm{~g} / \mathrm{dL})$ & 7.4 & 6.5 \\
\hline WBC $\left(4500-11,000 / \mathrm{mm}^{3}\right)$ & 1.3 & .7 & 11.3 \\
\hline PLT $\left(150,000-400,000 / \mathrm{mm}^{3}\right)$ & 78 & 41 & 3.0 \\
\hline Eosinophils $(1-3 \%)$ & 0.1 & 0.0 & 52 \\
\hline Immunoglobulins (upper limit 35\%) & 46 & 5.0 \\
\hline Spleen Size (7-14 cm) & 22 & 50 \\
\hline
\end{tabular}

Table 2. Comparison of Infectious Causes of Non-HIV associated Massive Splenomegaly

\begin{tabular}{|c|c|c|c|}
\hline & Visceral Leishmaniasis & Schistosomiasis \\
\hline $\begin{array}{c}\text { Constitutional symptoms: fever, night sweats, } \\
\text { weight loss }\end{array}$ & Present & $\begin{array}{c}\text { Acute: Present } \\
\text { Chronic: Typically asymptomatic }\end{array}$ \\
\hline WBC Count & Decreased & Increased \\
\hline Anemia & Present & Present \\
\hline Neutrophils & Decreased & No Change \\
\hline Eosinophils & Decreased & Increased \\
\hline Lymphadenopathy & Present & Absent \\
\hline Immunoglobulin & IgG & IgG and IgE \\
\hline Platelets & Decreased & Decreased \\
\hline
\end{tabular}

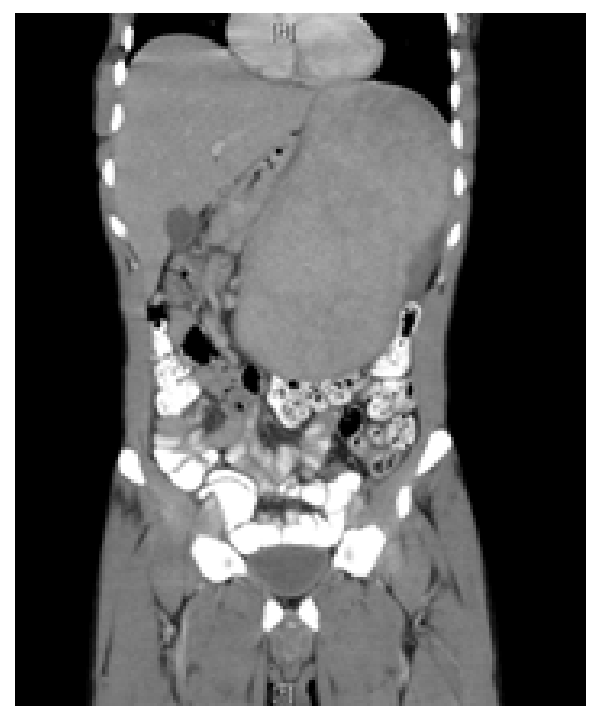

Figure 1. Abdominal CT of the patient on admission showing massive splenomegaly

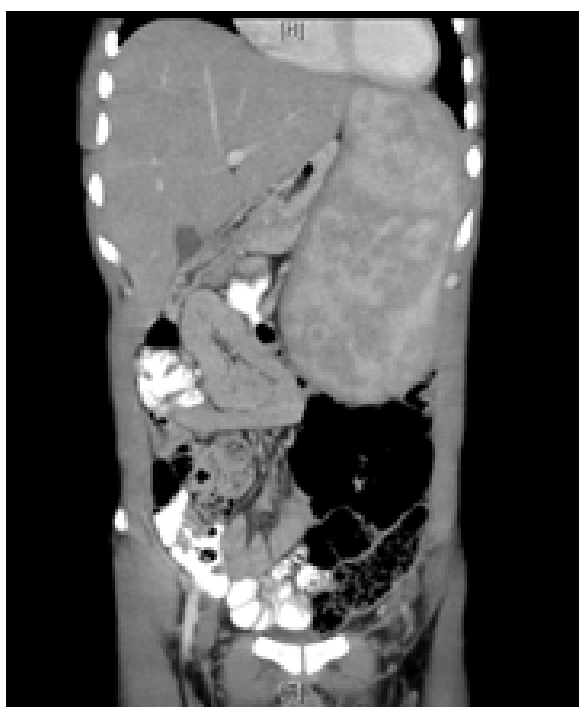

Figure 2. Abdominal CT of the patient 6 weeks after treatment. The spleen is still enlarged by to a lesser degree splenomegaly is due to replication and accumulation of the parasite in the reticuloendothelial system [2]. Nonspecific laboratory results of VL include anemia, neutropenia, eosinopenia, thrombocytopenia and hypergammaglobulinemia. The Leishmania of the patient was identified belonging to the Donovani complex using PCR methods. This complex encompasses both $L$. Donovani and $L$. Infantum specie. The patient came from East Africa, an area that is endemic for L. Donovani. Whether the infection was acquired in Eritrea or during his 9- month stay in a refugee camp in Ethiopia is unknown, as both countries are endemic for L. Donovani.

Massive spelenomegaly could also be due to Schistosomiasis. Splenomegaly in schistosomiasis can be due to two different pathological processes. In young children and adolescents, there is an inflammatory response as an early reaction to ova trapped in the perisinusoidal periportal spaces of the liver. There is additional nodular enlargement of the spleen. In adults, a longstanding intense infection causes massive deposition of diffuse collagen in the periportal spaces of the liver, leading to the pathognomonic periportal or Symmer's pipestem fibrosis. This fibrosis leads to portal hypertension and splenomegaly with collateral venous circulation [3].

Though our patient was diagnosed during the first day of hospitalization with S. Mansoni, which could allegedly explain the splenomegaly, the lack of significant liver involvement suggested an additional diagnosis. It is unknown which infection appeared first in the patient. Animal studies suggest prior infection with S. Mansoni allow subsequent coinfection with Leishmania to become more severe or persist longer than single Leishmania infection [4].

An additional infectious diagnosis that can explain massive splenomegaly is Hyperreacive Malarial Syndrome. This syndrome is caused by an aberrant immunological response to malaria due to the presence of elevated IgM antibodies against CD8 lymphocytes. This leaves a relatively unopposed effect of CD4 helper lymphocytes on B cells, which in turn causes reticuloendothelial hypertrophy and massive splenomegaly [5]. Our patient indeed lived most of his life in a place endemic to malaria, however he denied any history compatible with recurrent episodes of malaria. Additionally, his hypergammaglobulinemia consisted mostly of IgG rather than IgM.

To the best of our knowledge this is the first reported coinfection of $\mathrm{VL}$ and schistosomiasis infection in Israel. The exact pathological effect on each respective infection requires further research. 


\section{References}

1. Bern C (2012) Approach to the adult patient with splenomegaly and other splenic disorders. UptoDate, Waltham, MA Literature review current through: Jul 2012 .

2. Bern C (2013) Clinical manifestations and diagnosis of visceral leishmaniasis. UptoDate. In: Waltham MA (Ed.), Literature review current through: Mar 2013.
3. Gryseels B, Polman K, Clerinx J, Kestens L (2006) Human Schistosomiasis. The Lancet 368: 1106-18.

4. Abruzzi A, Fried B (2011) Coinfection of Schistosoma (Trematoda) with Bacteria, Protozoa and Helminths. Adv Parasitol 77: 1-85. [Crossref]

5. Puente S, Subirats M, Benito A, Rubio JM, Gonzalez-Lahoz JM (2001) Hyperreactive Malarial Splenomegaly in Europeans: Report of Five Cases. J Travel Med 8: 322-324. [Crossref]

Copyright: (2018 Paran Y. This is an open-access article distributed under the terms of the Creative Commons Attribution License, which permits unrestricted use, distribution, and reproduction in any medium, provided the original author and source are credited. 